\title{
Pocket-sized versus standard ultrasound machines in abdominal imaging
}

Ka Hei $\underline{T s}{ }^{1}$, MBChB, Wing Hang $\underline{L u k}^{1}$, FRCR, FHKAM, Mau Chu $\underline{\operatorname{Lam}}^{1}$, MBChB

ABSTRACT The pocket-sized ultrasound machine has emerged as an invaluable tool for quick assessment in emergency and general practice settings. It is suitable for instant and quick assessment in cardiac imaging. However, its applicability in the imaging of other body parts has yet to be established. In this pictorial review, we compared the performance of the pocketsized ultrasound machine against the standard ultrasound machine for its image quality in common abdominal pathology.

Keywords: abdomen, radiology, ultrasonography

\section{INTRODUCTION}

Ultrasonography is a valuable, noninvasive investigation tool. It is especially suitable for detecting abnormal fluid collection and pathology of abdominal organs. ${ }^{(1)}$ However, the standard ultrasound machine is too bulky and expensive for regular medical clinics, and instant access to an ultrasound facility is not always feasible. This has led to the development of smaller and more affordable ultrasound machines.

The first portable ultrasound machine was commercially available in 1975. (2) However, the battery-powered, pocketsized ultrasound machine did not arrive until the late 1990s. With recent advancements in technology, smaller and lighter devices with higher image quality are now available (such as those manufactured by GE Healthcare, Siemens and Philips). These pocket-sized ultrasound machines allow clinicians to have immediate visual correlation with physical examination findings. They also allow for quick and instant assessments, which are extremely important for emergency physicians. At a healthcare resource allocation level, they significantly reduce a patient's waiting time and improve clinician's workflow. Furthermore, the cost of pocket-sized ultrasound machines is much lower than that of standard ones (approximately USD 10,000 vs. USD 49,000). ${ }^{(3,4)}$ Hence, they are more likely to be acquired for use in general practitioners' clinics.

The pocket-sized ultrasound machine has been shown to be suitable for cardiac imaging in certain clinical settings. ${ }^{(5)}$ However, its applicability for the imaging of other body parts has yet to be established. In this pictorial essay, we compared the quality of images obtained from a pocket-sized ultrasound machine and that of a standard ultrasound machine in abdominal imaging. The pocket-sized ultrasound machine used was the GE Vscan (GE Healthcare, Wauwatosa, WI, USA) and the standard ultrasound machine used was the Philips iU22 (C5-2 Probe, iU22 Intelligent Ultrasound System; Philips, Bothell, WA, USA).

For the purpose of comparison, images of the same patient were used. Ultrasonographic examinations were completed in the same session using the standard, followed by the pocketsized, ultrasound machine. All ultrasonographic examinations were performed by a radiologist who has had more than ten years of experience in ultrasonographic imaging. The time taken for imaging using the two kinds of ultrasound machines was comparable.

In the first section of this article, we compare the normal anatomy of major abdominal organs using the standard and pocket-sized ultrasound machines. We then compared the images obtained using the standard ultrasound machine against those obtained using the pocket-sized ultrasound machine in common intra-abdominal pathologies.

\section{NORMAL ANATOMY IN THE ABDOMINAL REGION}

Pocket-sized ultrasound machines (with broad bandwidth phased array probe) provide satisfactory image quality for identification of most of the abdominal solid organs.

In Fig. 1, the spleen can be easily assessed from the left subcostal approach while the patient is in the left lateral decubitus position. The spleen is more echogenic than the liver and considerably more echogenic than the left renal cortex.

The kidney is a retroperitoneal organ. It can be imaged via the subcostal approach while the patient lies in a lateral decubitus position. The kidney shows a central echogenic renal sinus and a peripheral hypoechoic renal cortex. Between the sinus and cortex are several hypoechoic pyramids (Fig. 2).

The pancreas is difficult to visualise sonographically due to its size, location and echogenicity. It is best assessed via the anterior subxiphoid approach using the left lobe of the liver as an acoustic window. The echogenicity of the pancreas varies according to its fat content (Fig. 3).

The liver, being the largest solid organ within the abdomen, often presents with different pathologies. The left lobe can be imaged using the anterior subxiphoid approach in most patients. The right lobe should be imaged using both subcostal and intercostal approaches to optimise assessment (Fig. 4). 

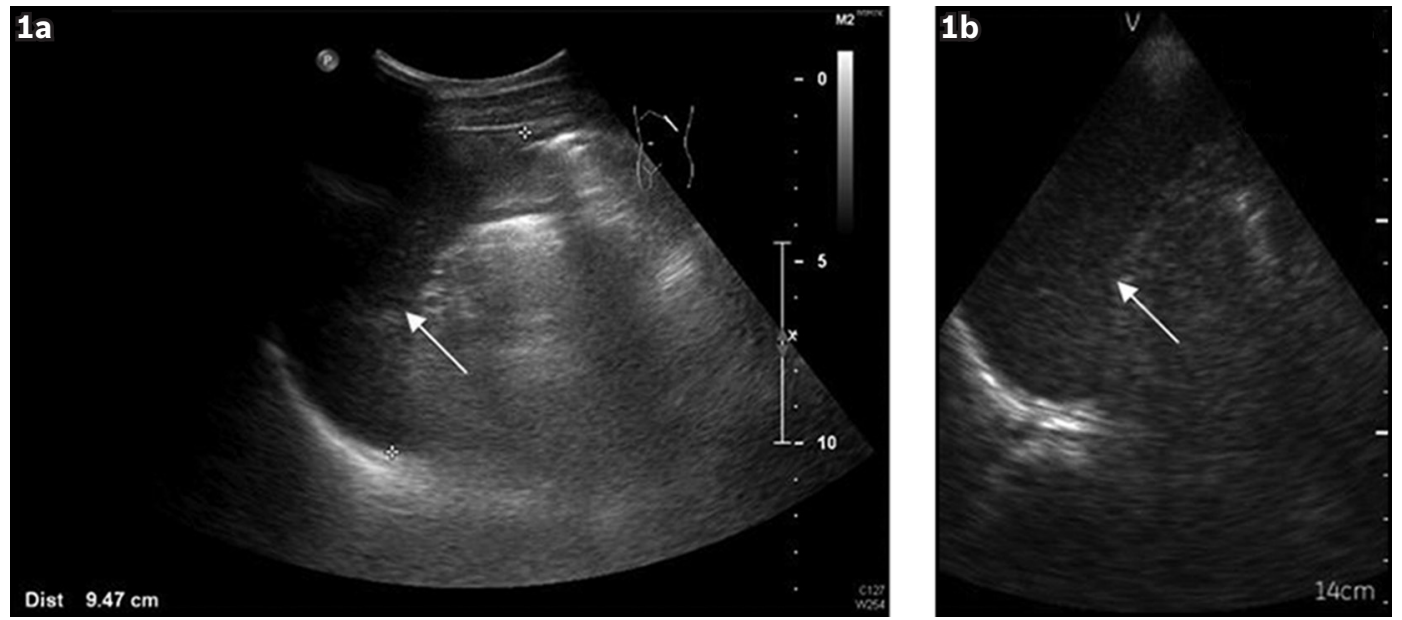

Fig. 1 Normal spleen (arrows). Images from (a) standard and (b) pocket-sized ultrasound machines.
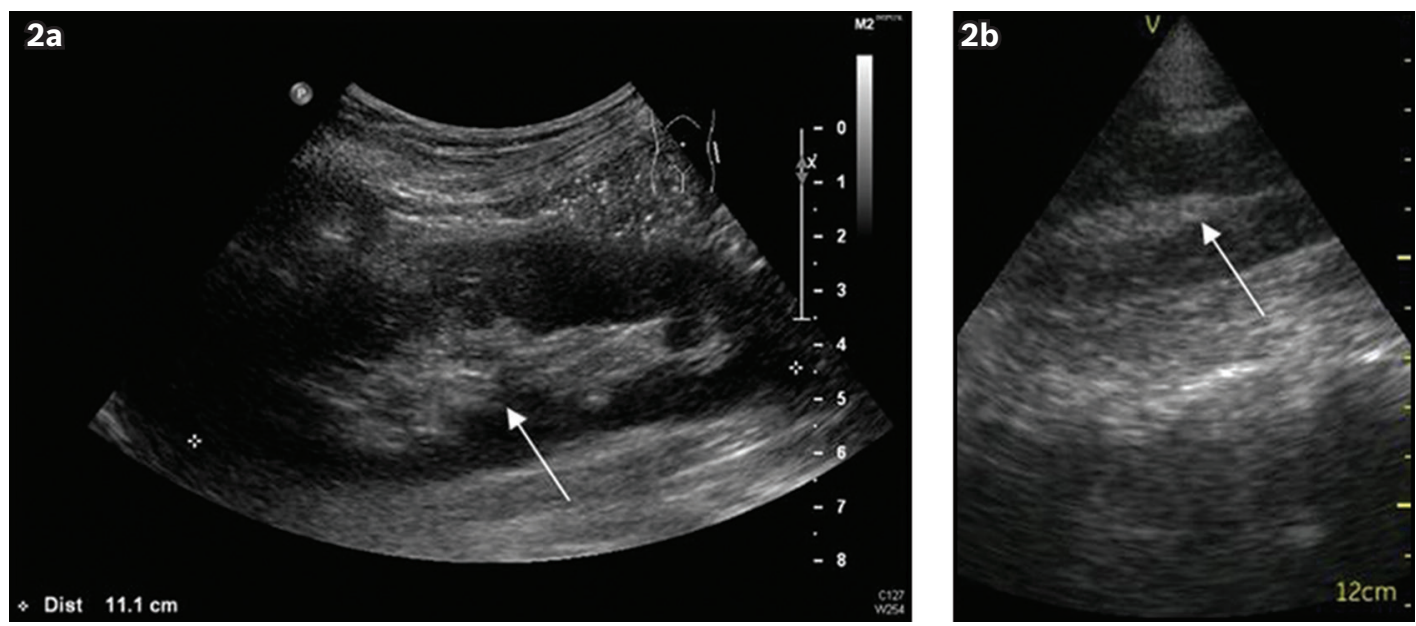

Fig. 2 Normal left kidney (arrows). Images from (a) standard and (b) pocket-sized ultrasound machines.
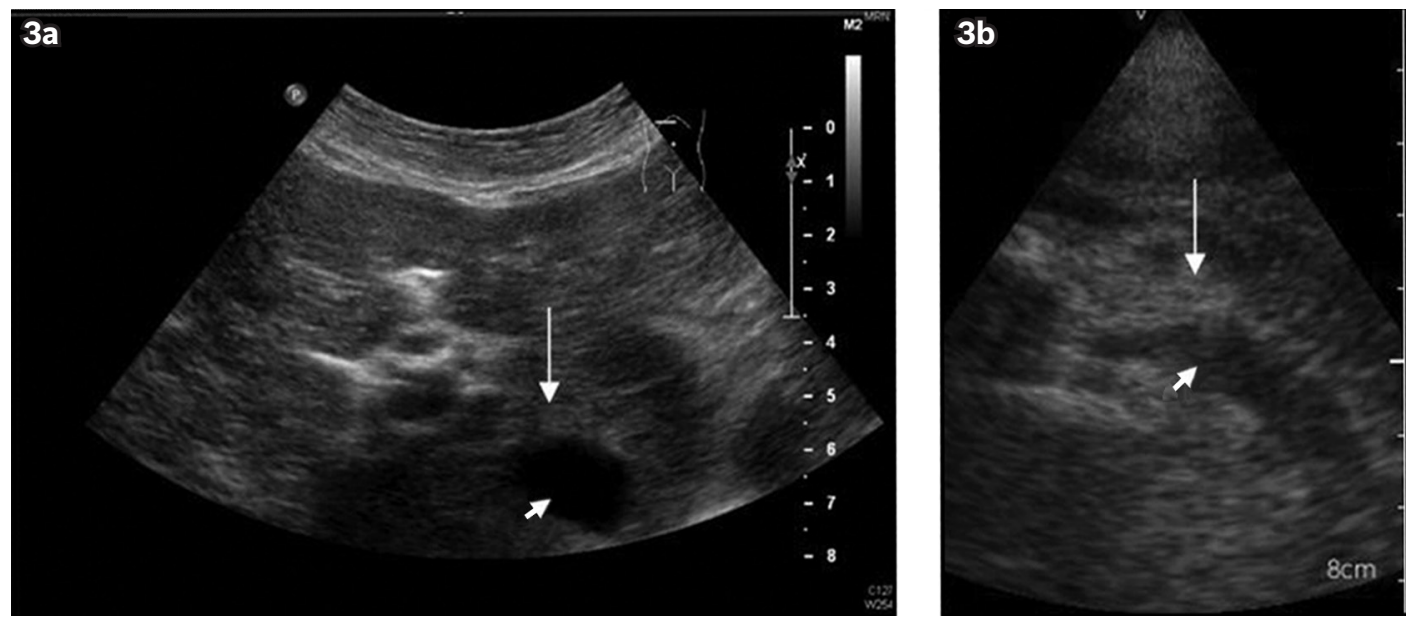

Fig. 3 Normal pancreas (arrows) and splenic vein (arrowheads). Images from (a) standard and (b) pocket-sized ultrasound machines. The pancreatic tail is not clearly seen.

The gallbladder is usually positioned beneath the liver and immediately adjacent to the interlobar fissure. Ideally, before imaging, the patient should fast for eight hours after midnight to ensure adequate gallbladder distension ${ }^{(6)}$ (Fig. 5).

The abdominal aorta, inferior vena cava and most large vessels can be identified as tubular hypoechoic structures at their expected locations. Colour Doppler is used in equivocal cases and also to help determine the flow direction (Figs. $6 \& 7$ ).

\section{ABNORMAL ULTRASONOGRAPHIC FINDING}

The pocket-sized ultrasound machine provides reasonable image quality in the identification of abdominal organs and major vessels. 

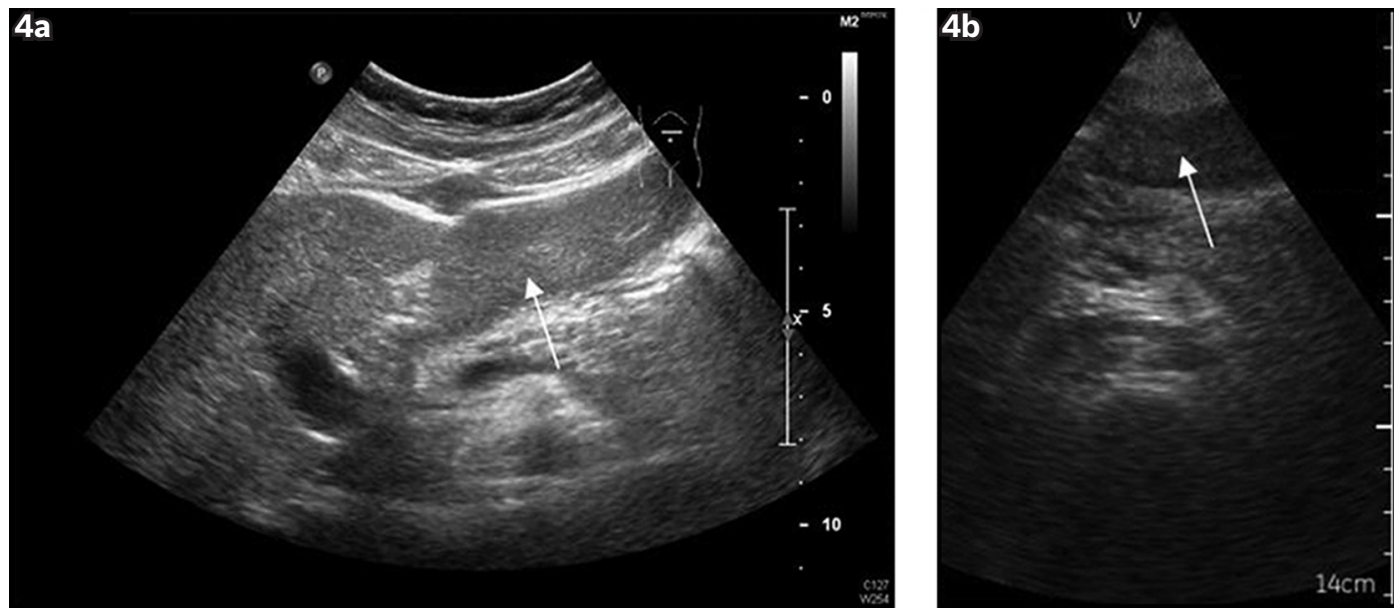

Fig. 4 Normal liver (arrows). Images from (a) standard and (b) pocket-sized ultrasound machines.
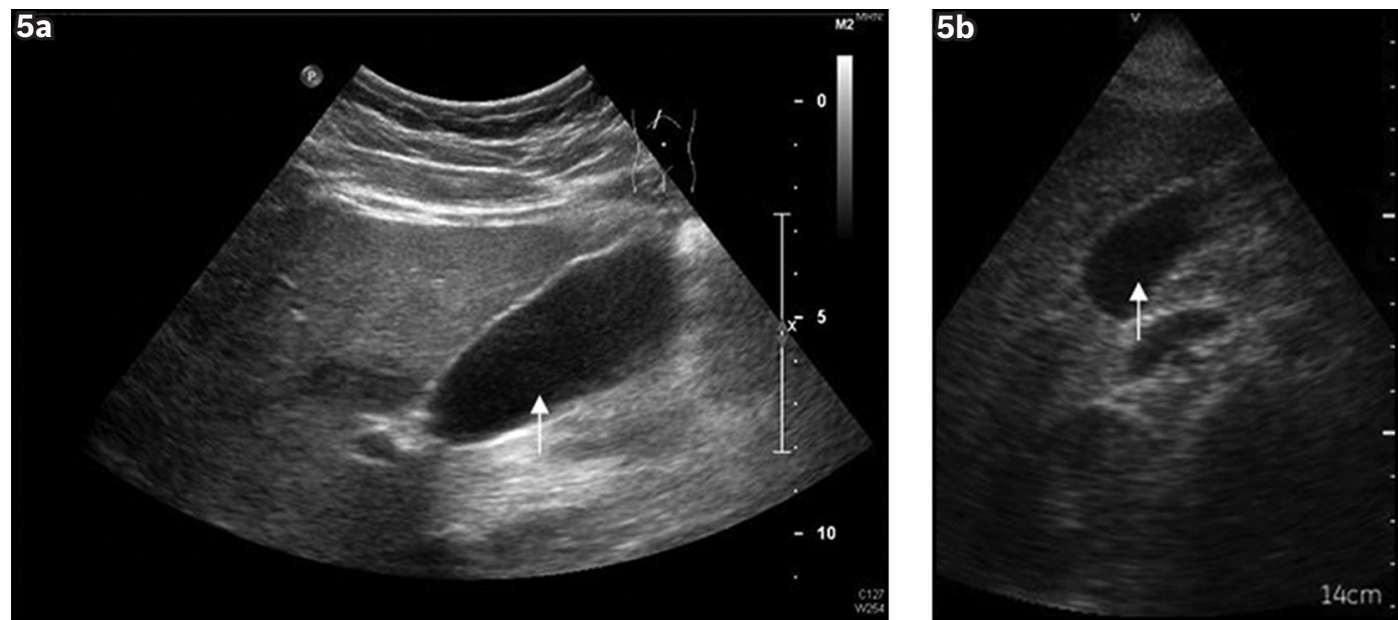

Fig. 5 Normal gallbladder (arrows). Images from (a) standard and (b) pocket-sized ultrasound machines.
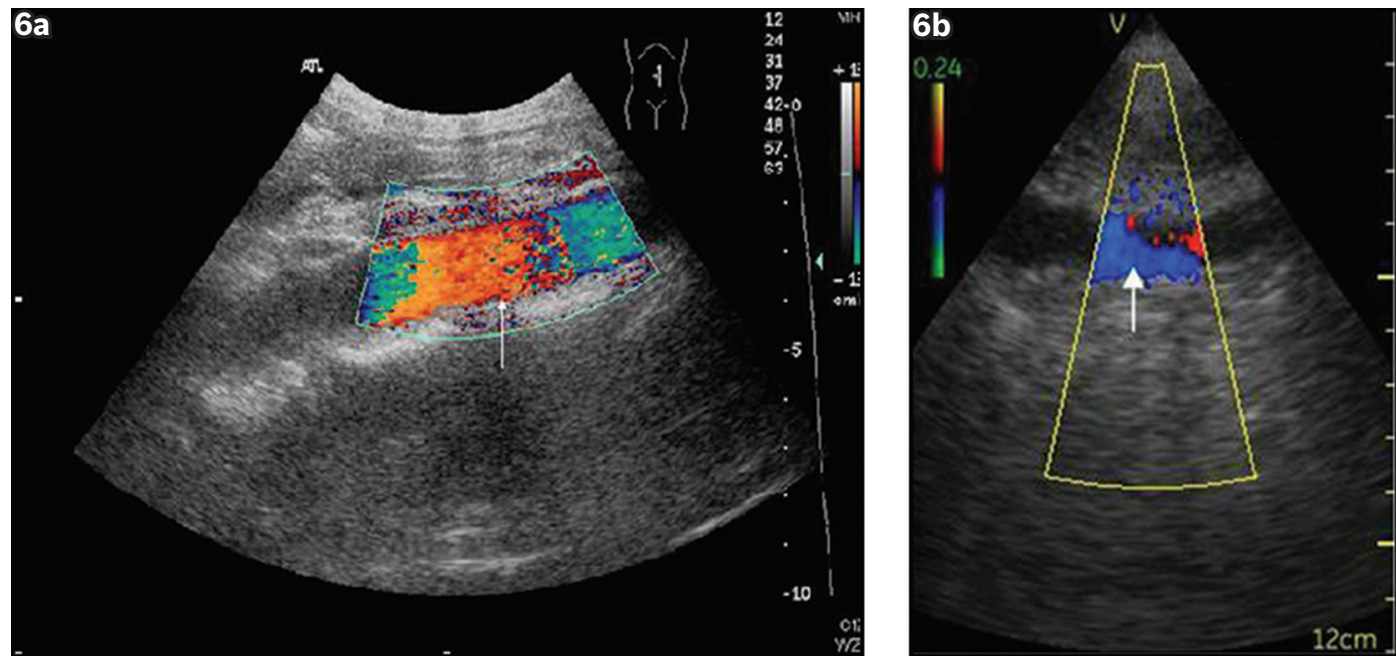

Fig. 6 Abdominal aorta (arrows). Images from (a) standard and (b) pocket-sized ultrasound machines.

We further analysed its suitability by comparing images acquired using standard and pocket-sized ultrasound machines in patients with common intra-abdominal pathologies. In certain clinical scenarios, such as detection of fluid and stones, the pocket-sized ultrasound machine provides acceptable image quality.

\section{Hydronephrosis}

Renal obstruction accounts for approximately $5 \%$ of patients with renal failure. Early detection helps prevent irreversible renal damage. Diagnosis of hydronephrosis relies on the detection of a dilated collecting system. It appears as anechoic regions 

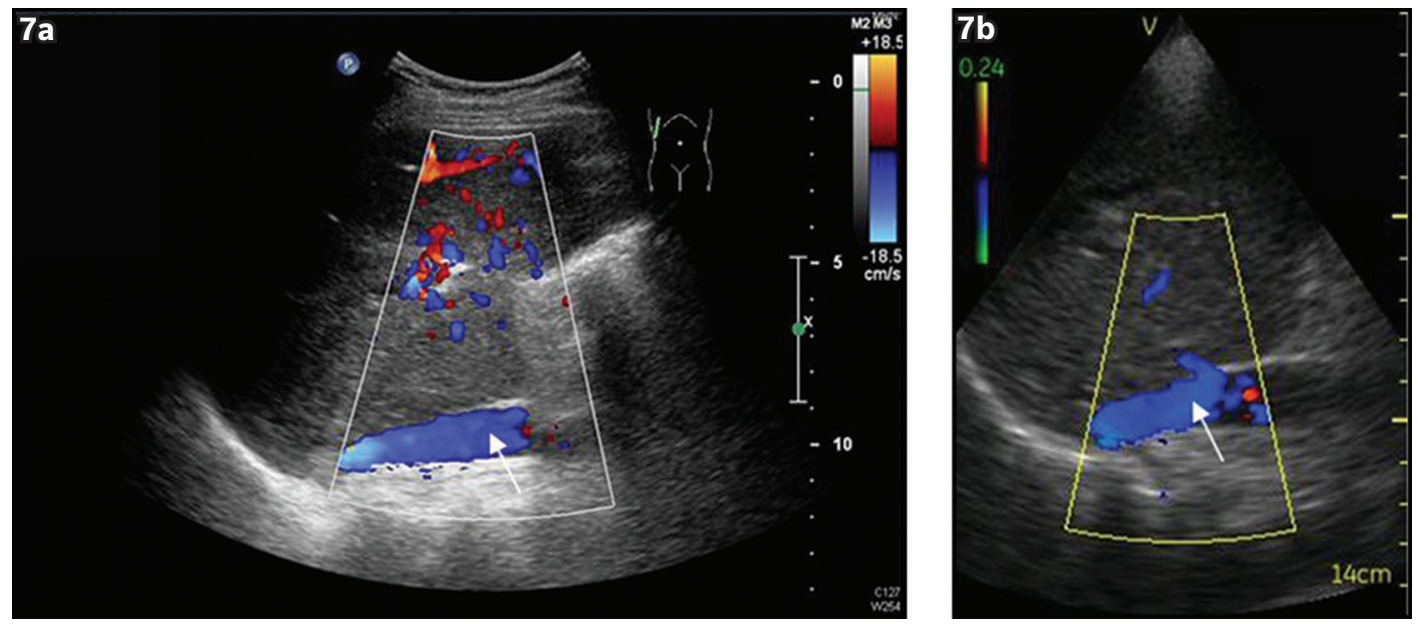

Fig. 7 Inferior vena cava (arrows). Images from (a) standard and (b) pocket-sized ultrasound machines.
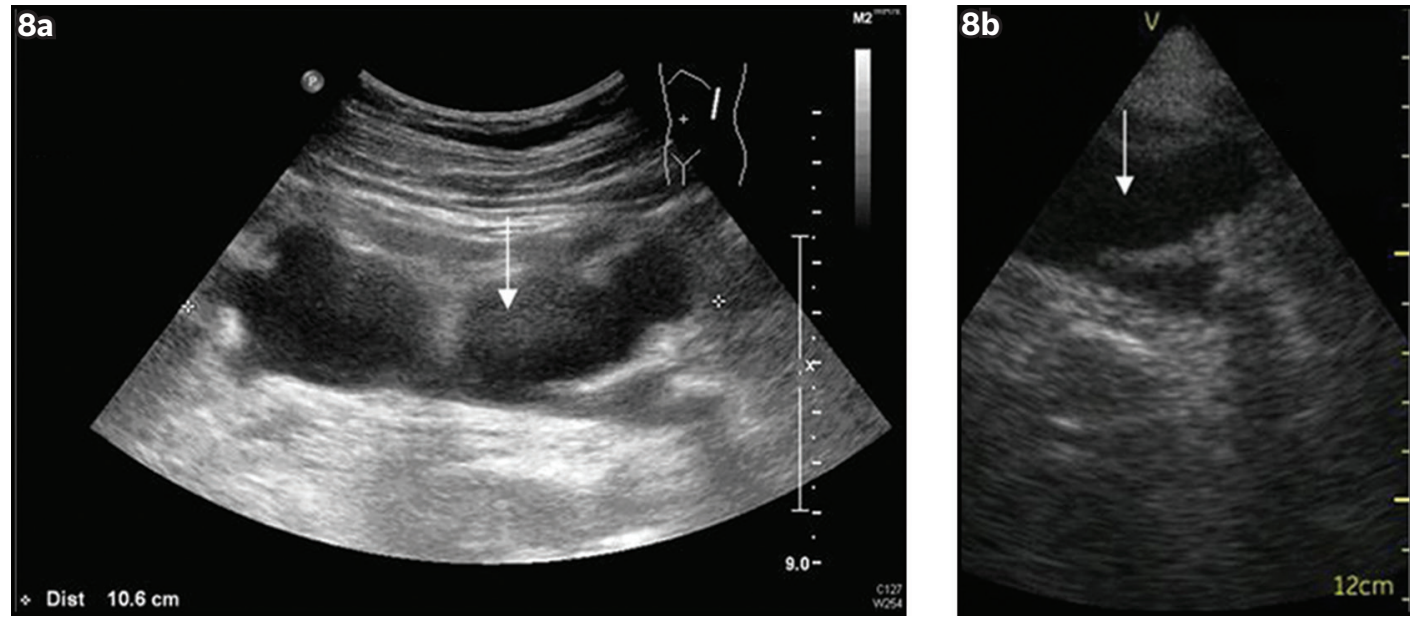

Fig. 8 Left hydronephrosis. (a) Image from the standard ultrasound machine shows a grossly dilated left calyceal system (arrows). (b) Image from the pocket-sized ultrasound shows an anechoic area at the expected site of the renal calices, suggesting hydronephrosis (arrows).
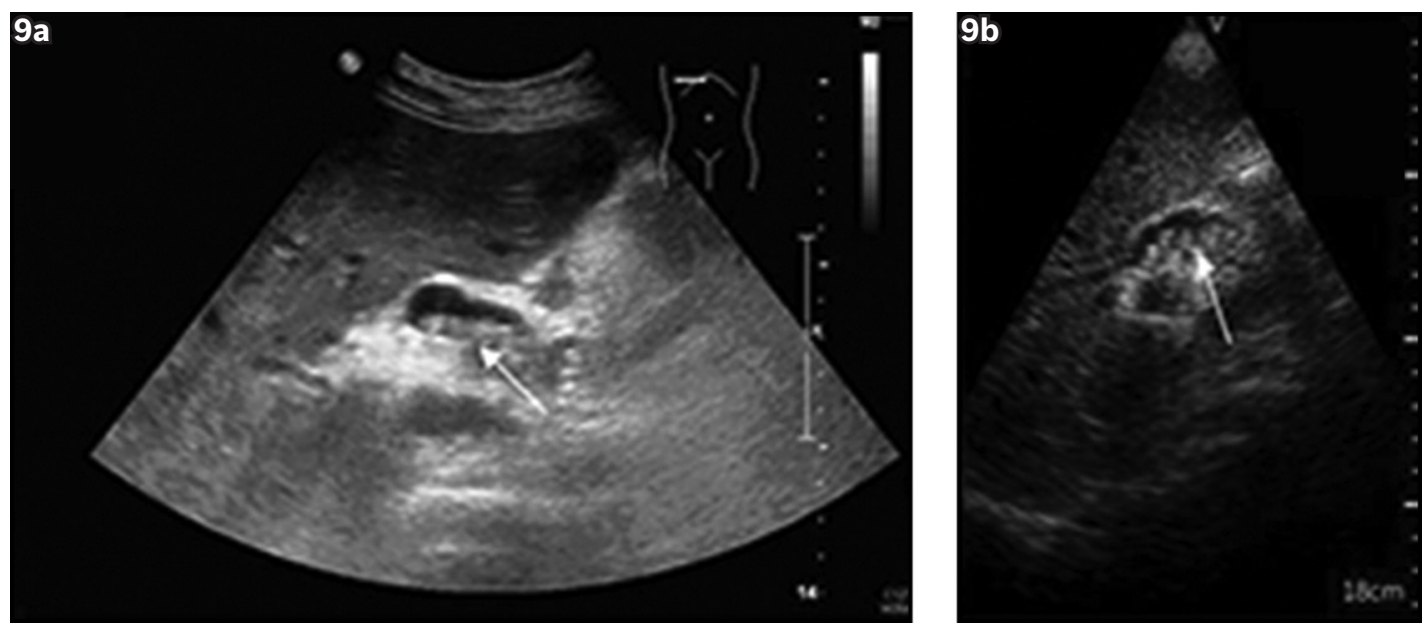

Fig. 9 Gallstones. (a) Image from the standard ultrasound machine shows mobile echogenic lesions (arrow) with posterior shadowing within the gallbladder, suggestive of gallstones. (b) Image from the pocket-sized ultrasound machine shows echogenic stones with posterior shadowing (arrow).

that conform to the expected site and shape of the renal calices (Fig. 8).

\section{Gallbladder and intrahepatic ductal stones}

Gallbladder stones are common, and a majority of them are asymptomatic. Among those that present with clinical symptoms, biliary colic is the most common. Ultrasonography is the most sensitive investigation tool for detecting gallstones. Gallstones appear as mobile intraluminal echogenic lesions with posterior acoustic shadowing $^{(7)}$ (Fig. 9). 

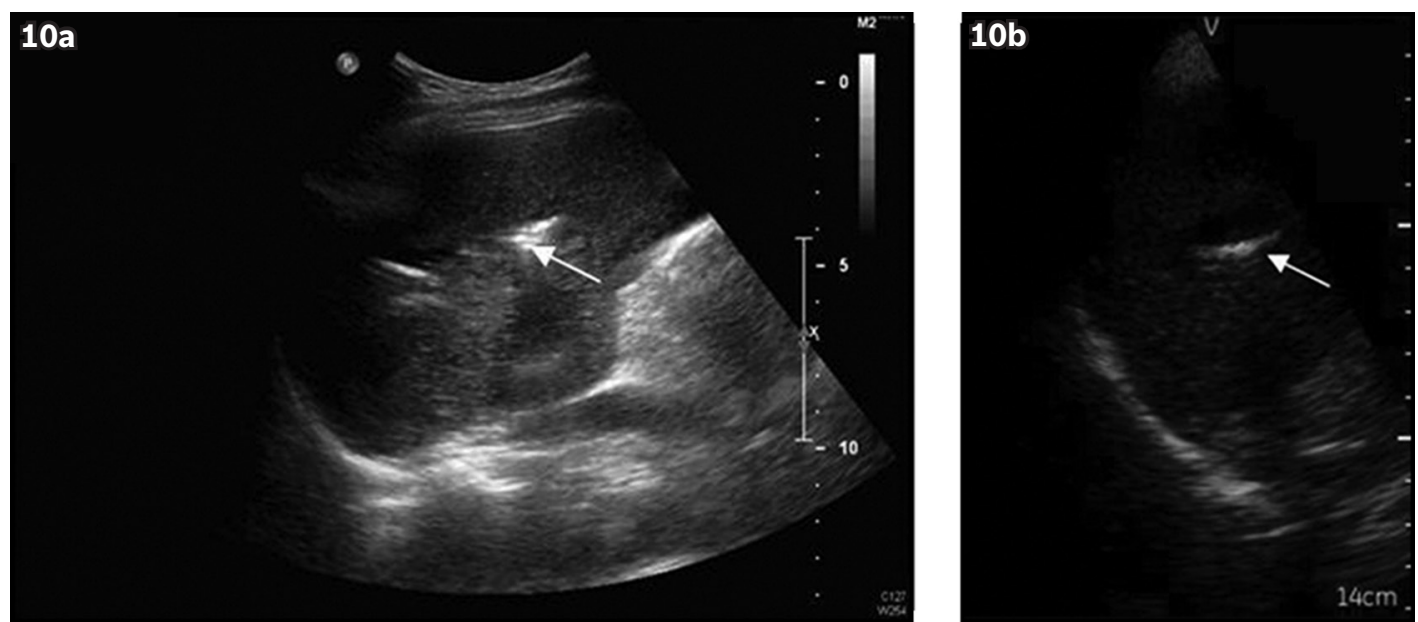

Fig. 10 Intrahepatic stones. (a) Image from the standard ultrasound machine shows linear echogenicity with posterior shadowing (arrow) within the liver parenchyma, which is suggestive of intrahepatic stones. (b) Image from the pocket-sized ultrasound machine shows similar-looking linear echogenicity with posterior shadowing (arrow).
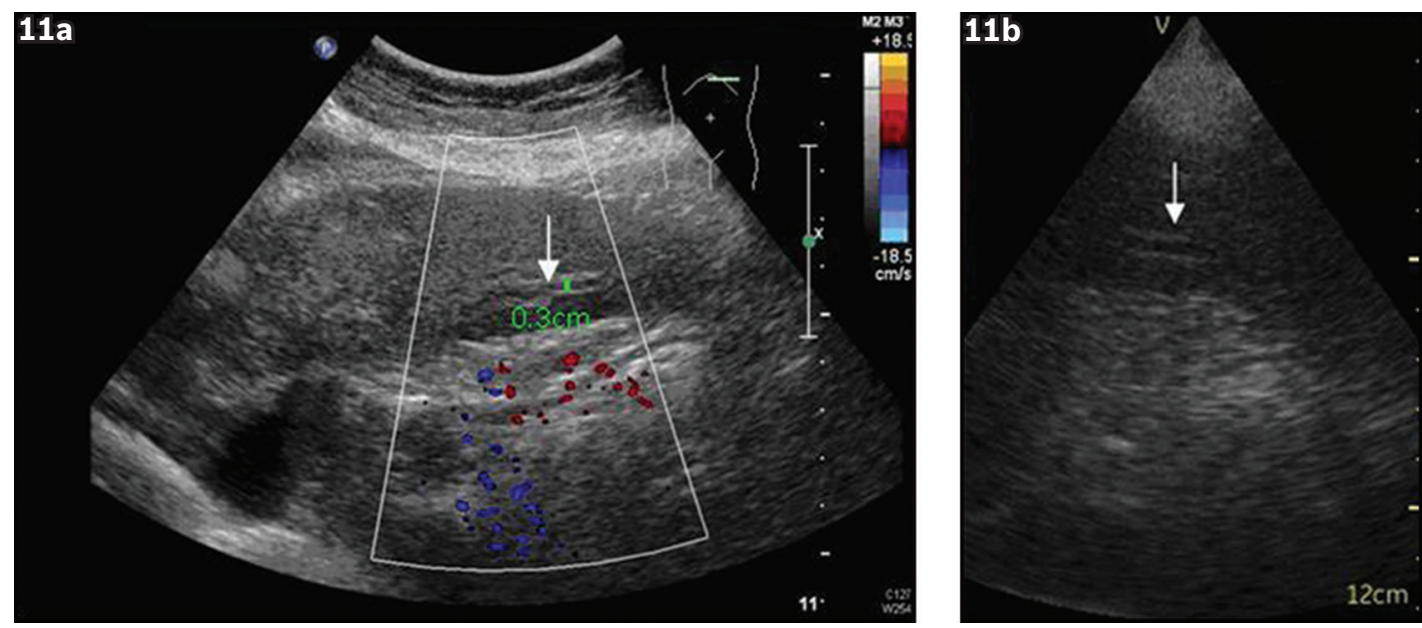

Fig. 11 Dilated left intrahepatic duct. (a) Image from the standard ultrasound machine shows dilated tubular structures without Colour Doppler signal (arrow), which is suggestive of intrahepatic ductal dilatation. (b) Image from the pocket sized ultrasound machine shows similar-looking dilated tubular structures (arrow).

Intrahepatic stones are another common finding, especially in patients with recurrent pyogenic cholangitis. It appears as linear echogenicity within the liver parenchyma, with or without coexisting ductal dilatation. Colour Doppler is effective in differentiating intrahepatic ducts from portal vein in equivocal cases $^{(8)}$ (Figs. $10 \& 11$ ).

\section{Intra-abdominal collection}

Early detection of intra-abdominal collection in septic patients with abdominal pain is crucial for deciding whether further intervention, such as surgical operation or percutaneous drainage, is required. It is identified as a loculated hypoechoic area with or without internal echogenicity outside the normal abdominal organs ${ }^{(9)}$ (Fig. 12).

\section{Abdominal aortic aneurysm}

Abdominal aortic aneurysm is not uncommon, especially in elderly patients with hypertension. Regular follow-up to check the aorta's diameter helps to guide appropriate intervention ${ }^{(10)}$ (Fig. 13). Both the pocket-sized and standard ultrasound machines can effectively identify the abdominal aorta.

\section{Pleural effusion}

Small amounts of loculated pleural fluids can be overlooked in conventional plain radiographs. Ultrasonography, on the other hand, is sensitive at detecting fluid, even trace amounts of effusion (Fig. 14). Both pocket-sized and standard ultrasound machines are able to detect pleural fluid.

\section{Renal and liver parenchymal disease}

Parenchymal disease has different causes and is commonly found in patients with deranged renal or liver function. Highresolution imaging is required to assess the echotexture of the solid organs in order to determine any parenchymal abnormality ${ }^{(11,12)}$ (Figs. 15 \& 16). Due to its reduced screen size and functionality, the pocket-sized ultrasound machine is inferior to standard ultrasound machine in assessing parenchymal echogenicity.

\section{Space-occupying lesion in organs}

Abscess and neoplasm are common pathologies found in intra-abdominal organs. However, accurate detection and characterisation of these lesions using ultrasonography can 

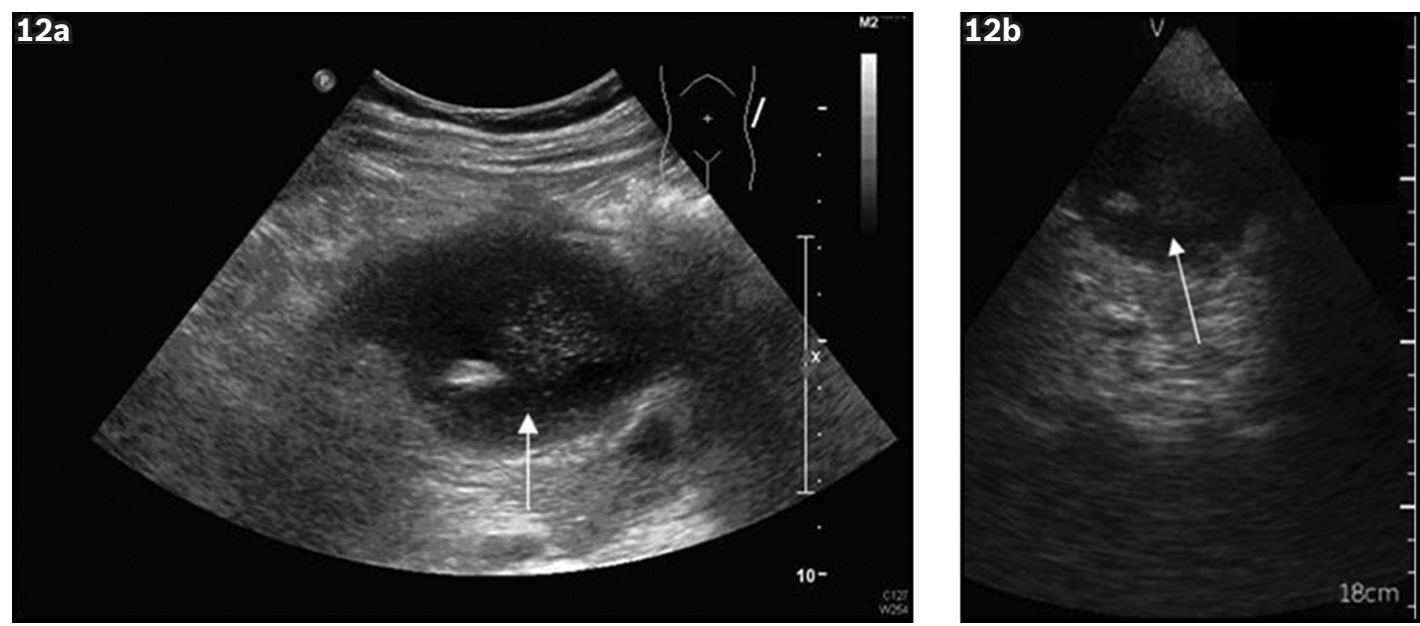

Fig. 12 Intra-abdominal collection. (a) Image from the standard ultrasound machine shows a loculated hypoechoic area with internal echogenicity (arrow), which is suggestive of collection. (b) Image from the pocket-sized ultrasound machine shows a similar-looking hypoechoic collection (arrow).
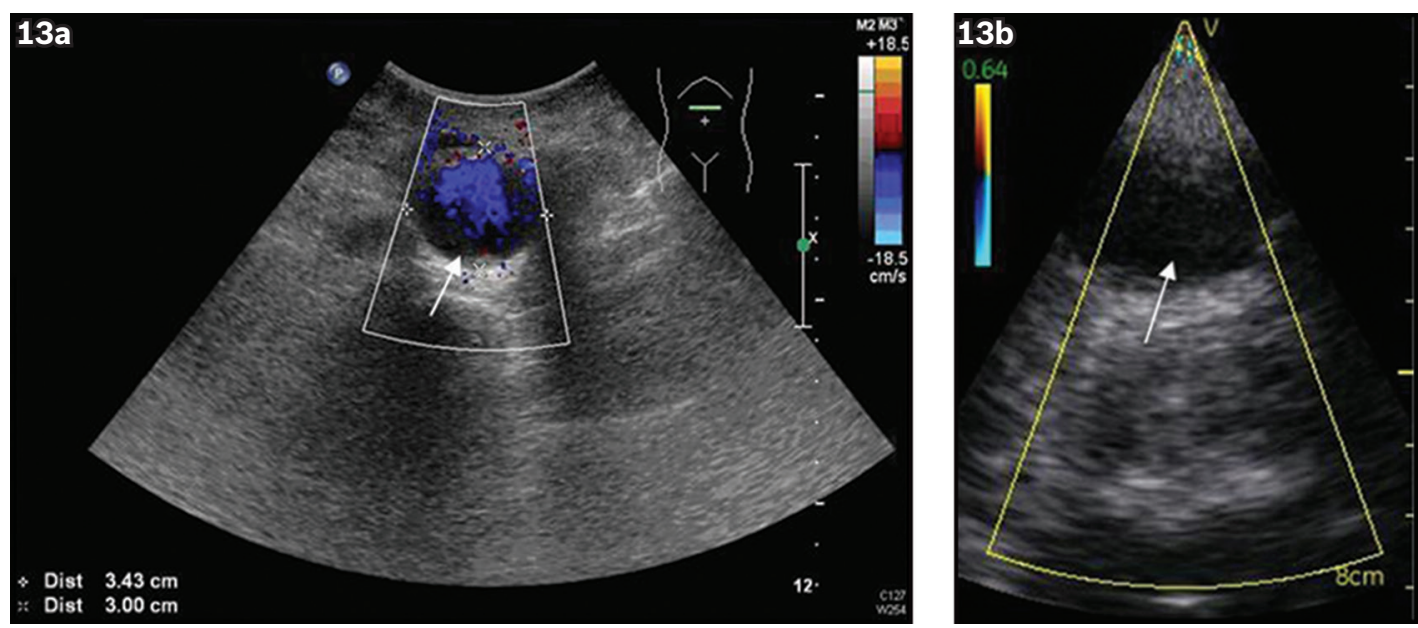

Fig. 13 Abdominal aorta aneurysm. (a) Image from the standard ultrasound machine shows a dilated abdominal aorta (arrow), which is suggestive of abdominal aorta aneurysm. (b) Image from the pocket-sized ultrasound machine shows comparable findings (arrow).
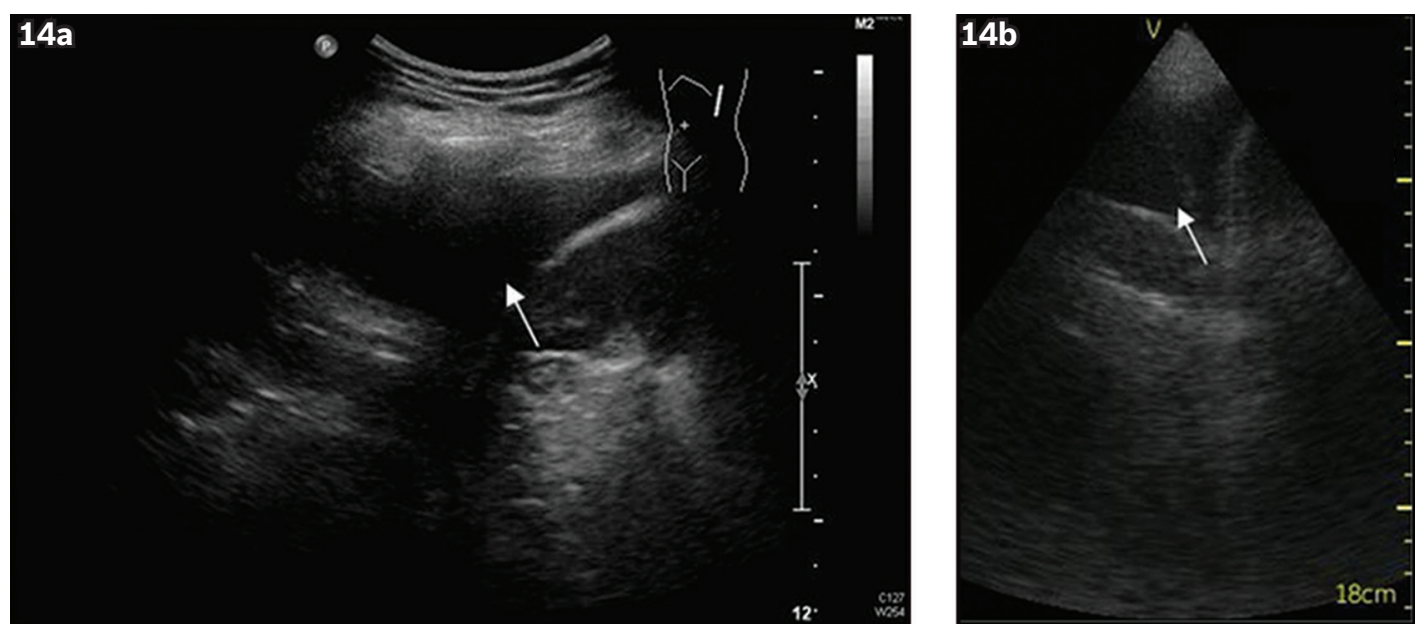

Fig. 14 Left pleural effusion. (a) Image from the standard ultrasound machine shows an anechoic fluid collection (arrow) just superior to left hemi-diaphragm, which is suggestive of effusion. (b) Image from the pocket-sized ultrasound machine shows similar findings (arrow).

sometimes be challenging, even for experienced radiologists. A high-resolution ultrasound machine is an invaluable tool for ensuring high sensitivity and specificity ${ }^{(13,14)}$ (Figs.17-19). Like parenchymal abnormality, examination of space-occupying lesion using a pocket-sized ultrasound machine is suboptimal compared to a standard ultrasound machine. 

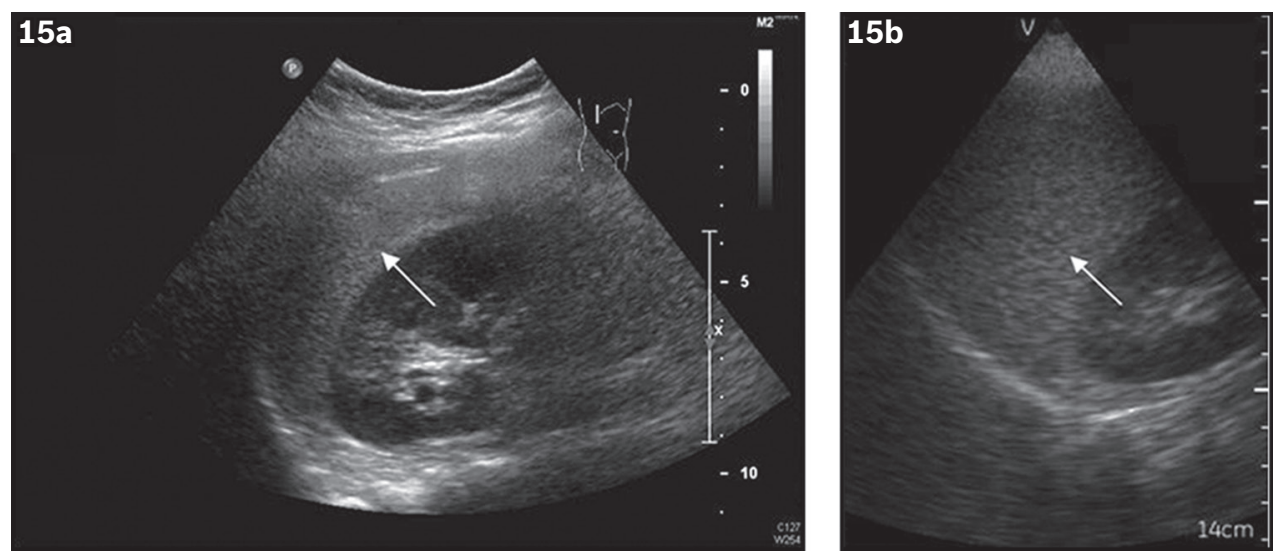

Fig. 15 Fatty liver. (a) Image from the standard ultrasound machine shows a diffuse increased hepatic echogenicity (arrow) compared to the renal cortex, which is suggestive of fatty changes. (b) Image from the pocket-sized ultrasound machine shows relative unremarkable liver echotexture (arrow), making diagnosis of fatty changes difficult.
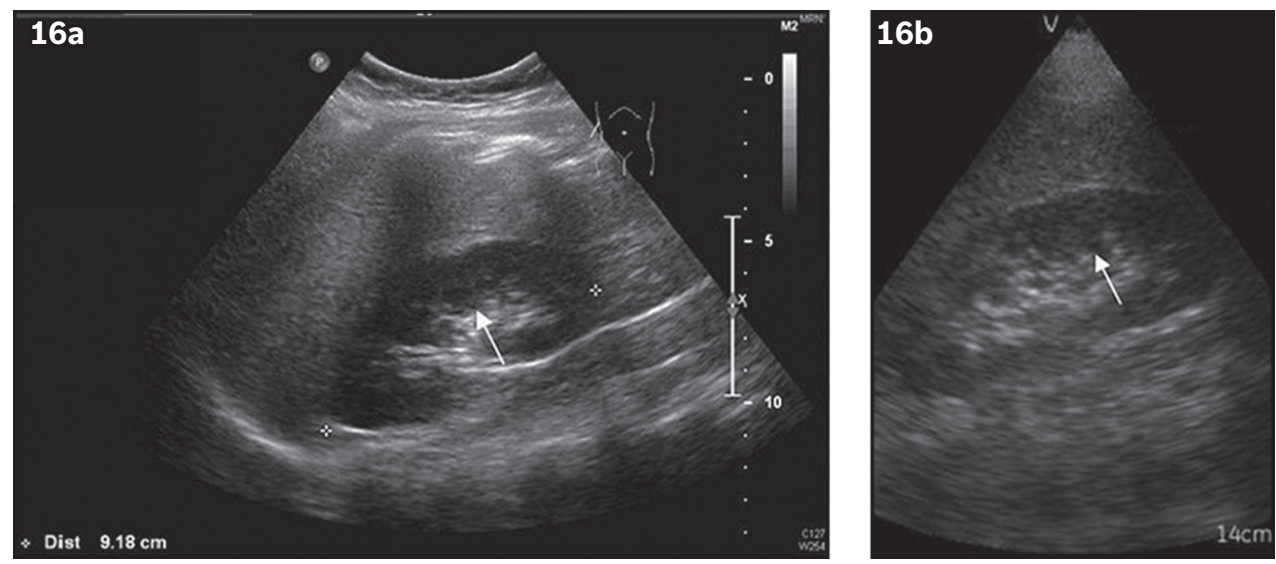

Fig. 16 Normal kidney. (a) Image from the standard ultrasound machine shows normal renal echotexture (arrow) with preserved cortical medullary differentiation. (b) Image from the pocket-sized ultrasound machine shows a mild increase in renal echogenicity (arrow) as compared with the renal medulla, resulting in false-positive findings.
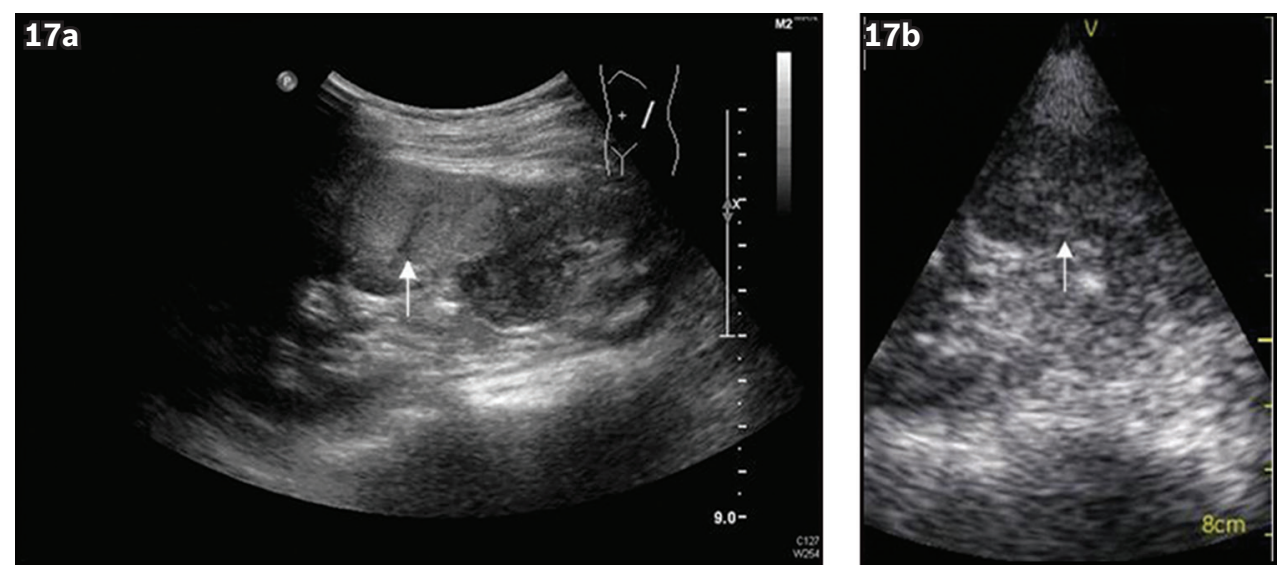

Fig. 17 Renal cell carcinoma. (a) Image from the standard ultrasound machine shows a heterogeneously hyperechoic lesion (arrow) arising from the middle pole of the left kidney, which is suggestive of renal cell carcinoma. (b) Image from the pocket-sized ultrasound cannot clearly identify the hyperechoic lesion (arrow).

\section{CONCLUSION}

Pocket-sized ultrasound machines are suitable for detecting a number of pathologies, such as hydronephrosis, gallstones, intrahepatic ductal stones and dilatation, intra-abdominal collection, major vessel abnormality such as abdominal aortic aneurysm, and fluid collection (pleural effusion or ascites), as shown in this article. Its satisfactory imaging ability is due to the high-contrast resolution difference of abdominal organs on ultrasonography. ${ }^{(15)}$ Therefore, pocket-sized ultrasound machines can be useful in emergency screenings. However, a major drawback is a narrowed field of vision and poor penetration, limiting its assessment of solid organ pathologies such as parenchymal disease and space-occupying lesions. Hence, thorough examination using a standard ultrasound machine is still required if solid organ pathologies are clinically suspected. 

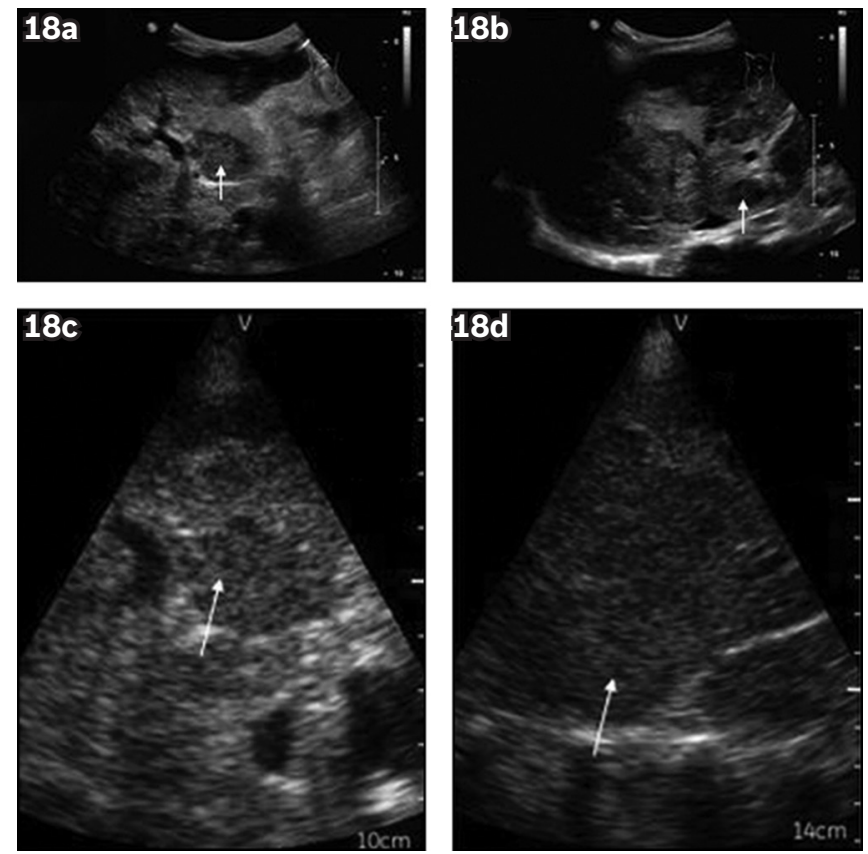

Fig. 18 Liver metastasis. Images (a) and (b), obtained from the standard ultrasound machine, show multiple hypoechoic lesions (arrows) scattered in both lobes of the liver, which is suggestive of metastasis. Images (c) and (d) from the same patient, obtained from the pocket-sized ultrasound machine, cannot clearly identify the hypoechoic lesions (arrows).
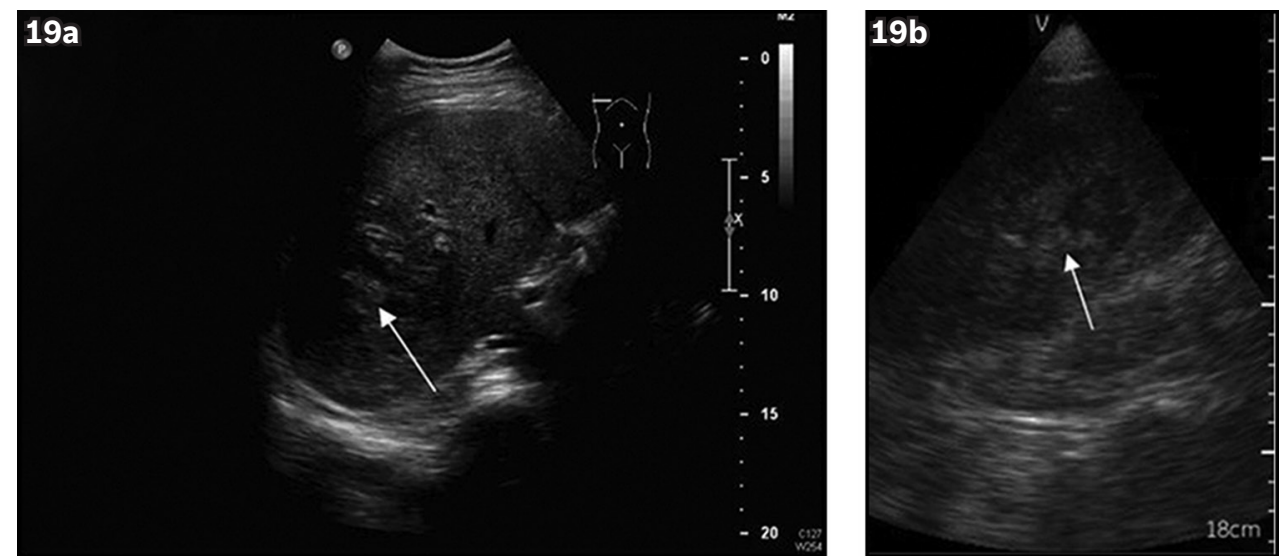

Fig. 19 Liver abscess. (a) Image from standard ultrasound machine shows a hypoechoic lesion (arrow) at the right lobe of the liver, suggestive of liver abscess. (b) Image from pocket-sized ultrasound machine cannot clearly identify the hypoechoic lesions (arrow).

\section{REFERENCES}

1. Ziskin MC. Fundamental physics of ultrasound and its propagation in tissue. Radiographics 1993; 13:705-9.

2. Woo J. A short history of the development of ultrasound in obstetrics and gynecology. Hong Kong, 2002 [online]. Available at: http://www.obultrasound.net/history2.html. Accessed September 2, 2012.

3. Pacific Medical Systems. Hong Kong, 2012 [online]. Available at: http:// www.vscan.com.hk. Accessed September 28, 2012.

4. Absolute Medical Equipment. United States of America, 2012 [online]. Available at: http://www.absolutemed.com/Medical-Equipment/UltrasoundMachines/Philips-IU22-Ultrasound-Machine. Accessed September 28, 2012.

5. Sicari R, Galderisi M, Voigt JU, et al. The use of pocket-size imaging devices: a position statement of the European Association of Echocardiography. Eur J Echocardiogr 2011; 12:85-7.

6. Turner MA, Fulcher AS. The cystic duct: normal anatomy and disease processes. Radiographics 2001; 21:3-22.

7. Costi R, Sarli L, Caruso G, et al. Preoperative ultrasonographic assessment of the number and size of gallbladder stones: is it a useful predictor of asymptomatic choledochal lithiasis? J Ultrasound Med 2002; 21:971-6. 8. Parulekar SG. Transabdominal sonography of bile ducts. Ultrasound Q 2000; 18:187-202.

9. Simon GL, Geelhoed GW. Diagnosis of Intra-abdominal Abscesses. A review. Am Surgery 1985; 51:431-6.

10. US Preventive Services Task Force. Screening for abdominal aortic aneurysm: recommendation statement. Ann Intern Med 2005; 142:198-202.

11. Tchelepi H, Ralls PW, Radin R, Grant E. Sonography of diffuse liver disease. J Ultrasound Med 2002; 21:1023-32.

12. Hamer OW, Anguirre DA, Casola G, et al. Fatty liver: imaging patterns and pitfalls. Radiographics 2006; 26:1637-53.

13. Daneman A, Navarro OM, Somers GR, et al. Renal pyramids: focused sonography of normal and pathologic processes. Radiographcis 2010; 30:1287-307.

14. Mortele KJ, Segatto E, Ros PR. The infected liver: radiologic-pathologic correlation. Radiographics 2004; 24:937-55.

15. Hangiandreou NJ. AAPM/RSNA physics tutorial for residents. Topics in US: B-mode US: basic concepts and new technology. Radiographics 2003; 23:1019-33. 


\section{SINGAPORE MEDICAL COUNCIL CATEGORY 3B CME PROGRAMME} (Code SMJ 201406A)

Question 1. Regarding ultrasonography in general:

(a) It involves non-ionising radiation.

(b) It is suitable for detecting abnormal gas collection.

(c) The advantage of ultrasonographic examination is its ability to correlate with physical examination findings.

(d) Doppler is especially useful for assessment of vascular structure.

Question 2. Regarding normal anatomy in abdominal ultrasonography:

(a) The normal echogenicity of the spleen is less echogenic than the liver.

(b) Renal sinuses of the kidney are echogenic.

(c) The patient should fast for two hours before imaging to ensure adequate distension.

(d) The echogenicity of the pancreas depends on its fat content.

Question 3. Regarding renal obstruction:

(a) It accounts for approximately $20 \%$ of patients with renal failure.

(b) It signifies irreversible renal damage.

(c) Hydronephrosis appears as anechoic regions that conform to the expected site and shape of the renal calices.

(d) Renal stones usually show posterior acoustic shadowing.

Question 4. Concerning the biliary system:

(a) Patients with recurrent pyogenic cholangitis are prone to developing common duct stone.

(b) Colour Doppler can help to differentiate dilated duct from portal vein.

(c) Gallbladder stones usually appear as fixed intraluminal echogenic lesions.

(d) Intrahepatic duct stones must be associated with ductal dilatation.

Question 5. In assessing a fluid-filled structure:

(a) The presence of intra-abdominal collection in a septic patient would not alter management options.

(b) An abscess is usually identified as a loculated homogeneous hypoechoic area.

(c) Ultrasonography can assess the size of the abdominal aorta accurately.

(d) Plain radiography is superior to ultrasonography in detecting pleural effusion.

\section{Doctor's particulars:}

Name in full

MCR number

Specialty:

Email address

\section{SUBMISSION INSTRUCTIONS:}

(1) Log on at the SMJ website: http://www.sma.org.sg/publications/smjcurrentissue.aspx and select the appropriate set of questions. (2) Provide your name, email address and MCR number. (3) Select your answers and click "Submit".

\section{RESULTS:}

(1) Answers will be published in the SMJ August 2014 issue. (2) The MCR numbers of successful candidates will be posted online at the SMJ website by 4 August 2014. (3) Passing mark is $60 \%$. No mark will be deducted for incorrect answers. (4) The SMJ editorial office will submit the list of successful candidates to the Singapore Medical Council. (5) One CME point is awarded for successful candidates. 